\title{
First Dates: El Proyecto y la Teoría
}

\author{
Agrasar Quiroga, Fernando; Paz Agras, Luz; García Requejo, Zaida
}

Universidade da Coruña, ETS de Arquitectura

\section{RESUMO}

La metodología docente que se expone en este escrito ha sido aplicada en las clases prácticas de la asignatura Teoría de la Arquitectura del sexto semestre del Grado en Estudios de Arquitectura de la Escuela Técnica Superior de Arquitectura de la Universidad de A Coruña durante el curso 2016-17. El ejercicio se basa en el Aprendizaje Basado en Proyectos (ABP), con el objetivo de que los estudiantes comprendan los contenidos de teoría de la arquitectura como una herramienta necesaria y eficaz a la hora de proyectar. El planteamiento es el siguiente: el profesorado selecciona y propone la lectura de un texto teórico relevante a cada grupo, que está seleccionado del material de temario de la asignatura, en el que tienen un especial peso las aportaciones teóricas comprendidas entre las manifestaciones tempranas de las vanguardias y el fin de la hegemonía del Movimiento Moderno; los estudiantes deben establecer una relación entre la lectura de las ideas fundamentales del texto sobre un edificio de otro autor, de manera que puedan elaborar una ligazón entre ideas teóricas y proyecto arquitectónico; una vez aceptada la elección del edificio, su análisis se lleva a cabo desde las ideas fundamentales del texto asignado utilizando las herramientas gráficas, propias de nuestra disciplina. En este proceso, los resultados son abiertos e impredecibles, aunque siempre fruto de una aproximación rigurosa.

PALABRAS CLAVE: Teoría, proyecto, crítica, Arquitectura Moderna, Arquitectura Contemporánea 


\section{CITA RECOMENDADA:}

Agrasar Quiroga, F., Paz Agras, L., García Requejo, Z. (2018). First Dates: El Proyecto y la Teoría. En E. de la Torre Fernández (ed.) (2018). Contextos universitarios transformadores: retos e ideas innovadoras. II Xornadas de Innovación Docente. Cufie. Universidade da Coruña (pág. 245-260).

DOI capítulo: https://doi.org/10.17979/spudc.9788497496780.245

DOl libro: https://doi.org/10.17979/spudc.9788497496780

\section{ABSTRACT}

The methodology exposed on this paper has been applied in the subject of Theory of Architecture in the sixth semester of the Degree in Architecture of the School of Architecture in the University of A Coruña during the 2016-17 course. The exercise is based on Project-Based Learning (ABP) and the objective is that students understand the content of architectural theory as a necessary and effective tool when designing. The approach is as follows: professors select and propose the reading of a relevant theoretical text to each group, selected from the contents of the course. Theoretical contributions between the early manifestations of the Avant-Garde and the end of the hegemony of the Modernism have special importance in these texts. Students must establish a relationship between fundamental concepts from the text and a building by another author, they propose, to make connections between theoretical ideas and an architectural project. Once the choice of the building is accepted, its analysis is carried out from fundamental ideas of the assigned text using graphic tools that are typical of our discipline. In this process, results are open and unpredictable although they are always the result of a rigorous approach.

KEY WORDS: Theory, Project, Criticism, Modern Architecture, Contemporary Architecture. 


\section{INTRODUCCIÓN}

La tradicional división de saberes en teoría y práctica, consagrada desde la tratadística clásica, resulta especialmente empobrecedor en la enseñanza de la arquitectura. Si entendemos el núcleo de la capacidad disciplinar como el proceso creativo de proyecto, en el que cristalizan todos los saberes propios de la arquitectura, la separación metodológica del mundo de las ideas del ámbito de la experiencia creativa es un error a evitar. Es habitual que el estudio de la teoría de la arquitectura se acometa desde un punto de vista histórico, que, en la mayoría de los casos, es percibida por el alumno como una sucesión de hallazgos teóricos, vinculados a un tiempo y unas circunstancias que no tienen nada que ver con la contemporaneidad. Al mismo tiempo, en la práctica proyectual académica se exploran experiencias de interés, pero a través de referencias parciales y vinculadas a las circunstancias del programa planteado. Conscientes de este problema pedagógico, el objetivo que se plantea esta nueva metodología docente de la asignatura de Teoría de la Arquitectura es precisamente trazar una estrategia que permita alcanzar un perfecto acuerdo entre teoría y trabajo creativo, impulsando aquellos aspectos que puedan dotar al alumno de criterios necesarios a la hora de acometer su práctica proyectual.

\section{DESCRICIÓN DE LA EXPERIENCIA}

Esta metodología docente ha sido aplicada en las clases prácticas de la asignatura Teoría de la Arquitectura del sexto semestre del Grado en Estudios de Arquitectura de la Escuela de Arquitectura de la Universidade da Coruña durante el curso 2016-17. Forma parte de un conjunto de estrategias, en las que se viene trabajando desde cursos anteriores, dirigidas hacia la exploración de los vínculos entre Teoría y Proyecto.

En el tercer año del grado en arquitectura los alumnos han adquirido conocimientos básicos sobre diversas materias: herramientas de representación, primeras nociones de cálculo estructural, principios básicos de construcción, etc. 
En el ámbito de la Composición Arquitectónica, al que pertenece Teoría de la Arquitectura, han cursado una asignatura introductoria, el primer año, y una de historia del arte, el segundo, mientras que en proyectos han podido asistir a dos cursos con un nivel acorde a iniciación y primeras propuestas. Se trata, por tanto, de un momento en el que el alumno se ha enfrentado a proyectos arquitectónicos de escala reducida, que le han permitido ser consciente de la complejidad del proceso y que genera la curiosidad por el conocimiento de obras arquitectónicas que puedan ser usadas como referencias para sus propias propuestas.

En este sentido, la estrategia docente pasa por hacer ver al alumno la teoría como una herramienta PROPOSITIVA, a partir de la cual poder basarse para desarrollar su propia idea de arquitectura, junto con su propia experiencia vital y todo el amplio abanico de conocimientos disciplinares. Se huye, por tanto, de la acumulación de datos con un mero interés en sí mismo, para tratar de incidir en un aprendizaje creativo que ponga su acento en las relaciones transversales entre los conocimientos adquiridos por el estudiante. De este modo, se prioriza el trabajo reflexivo, que permite al alumnado adoptar una actitud activa en el proceso de aprendizaje, frente a la tradicional clase magistral, que sigue formando parte del proceso de aprendizaje. Esta actitud activa hace que se debilite el carácter impositivo de verdades incuestionables por parte del profesorado, dando paso al valor pedagógico de la incertidumbre. El ejercicio se realiza en grupos de tres alumnos, apostando por el trabajo en equipo para general un mayor grado de reflexión y debate. Durante el horario presencial de las clases prácticas, los grupos van exponiendo el avance de sus planteamientos a toda la clase, de modo que todos participan de sus reflexiones y hallazgos, al tiempo que los profesores orientan el desarrollo del trabajo.

El hecho de poner en contacto, en una primera aproximación intuitiva, un texto teórico y un edificio, cuya relación no es evidente, es el motivo por el que se ha bautizado a esta propuesta con el nombre "First Dates", ya que al ahondar en las diferentes posibilidades de conexiones entre ambos, es posible comprender el vínculo imprescindible existente entre ideas y obras 
arquitectónicas. Si, por el contrario, la pareja de edificio-texto elegida no resulta especialmente interesante, el fracaso tiene, también, un alto valor pedagógico.

La elaboración de este trabajo práctico permite a los alumnos ahondar en los siguientes puntos:

- Interrelacionar textos teóricos (ideas) y obras construida.

- Utilizar el conocimiento de la teoría de la arquitectura como mecanismo de juicio en los procesos de renuncias y elecciones.

- Comprender el proceso proyectual como el resultado de renuncias y elecciones.

- La explicación del proyecto como intención arquitectónica.

- La explicación del proyecto como concreción teórica.

\section{RESULTADOS}

A continuación se describen algunos de los trabajos desarrollados dentro de esta propuesta educativa, organizados en función de las diferentes estrategias empleadas por los distintos equipos de alumnos.

UN MISMO AUTOR, DISTINTOS ENFOQUES

Los tres primeros pares de trabajos aquí expuestos muestran diferentes aproximaciones por parte de los alumnos a la hora de enfrentarse a textos pertenecientes al mismo autor.

En el primer caso, si bien el texto analizado no es el mismo, sí lo es su autor, Bruno Taut. Ambos equipos se valen de un edificio construido por el autor del texto, el Pabellón de Cristal de la Exposición de la Werkbund de 1914, a la hora de acometer el análisis comparativo con la propuesta arquitectónica escogida por cada uno de ellos. En la primera aproximación (Figura 1), Ios alumnos extraen características que consideran definitorias de este pabellón para poder aplicarlas al edificio de la Mediateca de Sendai de Toyo Ito. En el segundo trabajo (Figura 2), los alumnos pretender buscar una respuesta contemporánea equivalente a ese pabellón de vidrio de principios del siglo XX. Así, optan por analizar el Pabellón de la Serpentine propuesto 
por el estudio BIG, destacando las necesarias diferencias con respecto al original, derivadas del correspondiente contexto temporal.

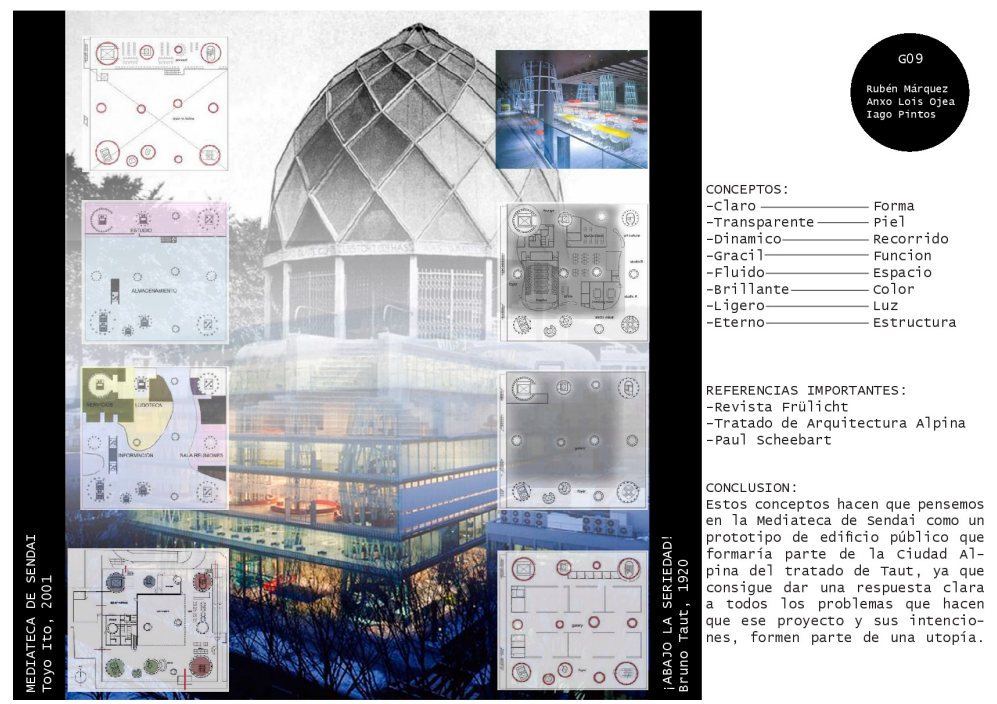

Figura 1. Análisis comparativo entre el texto "Abajo la Seriedad", de Bruno Taut y la Mediateca de Senday, de Toyo Ito.
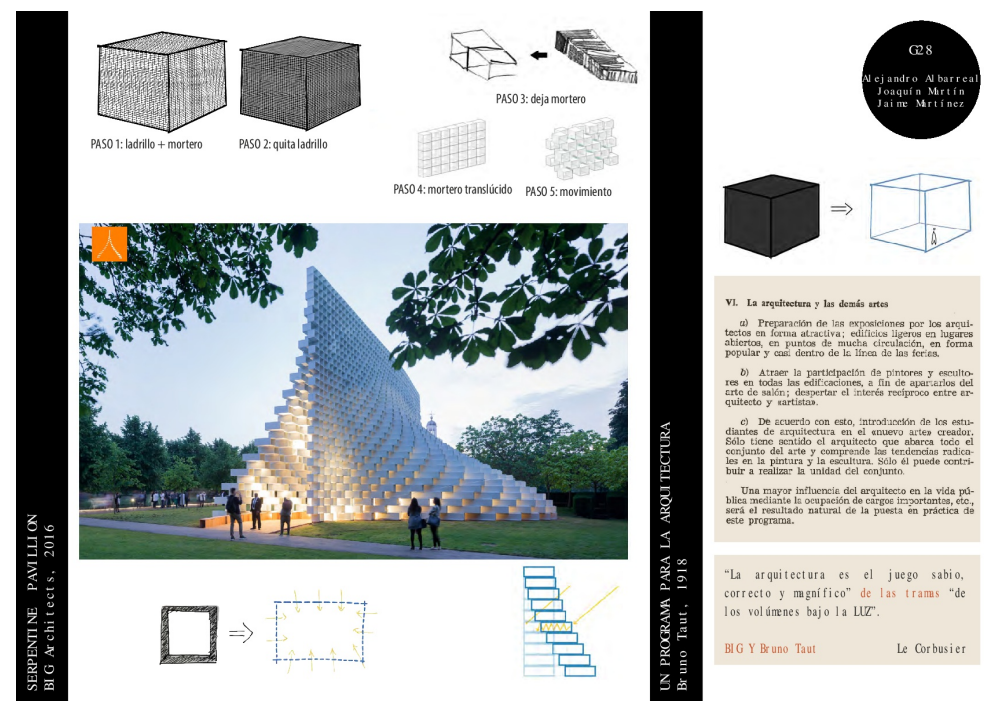

Figura 2. Análisis comparativo entre el texto "Un programa para la arquitectura", de Bruno Taut y el Serpentine Pavilion, de BIG Architects. 
De un modo similar, a la hora de analizar el texto de los autores Pichler y Hollein, titulado "Arquitectura Absoluta", el primero de los grupos aquí mencionados escoge el "edificio máquina", por considerarlo representativo de las ideas del texto (Figura 3). Así, acomete un análisis del Blur Building, diseñado por los arquitectos Diller \& Scofidio buscando identificarlo como la materialización de lo descrito en el texto. En el segundo caso (Figura 4), de modo similar a lo propuesto por el primer trabajo anteriormente expuesto, se extraen conceptos del texto para buscar su correspondencia en la construcción de la Dance House de Frank Gehry. Este trabajo se ha desarrollado dentro del marco Teaching in English que lleva a cabo la Escuela de Arquitectura.
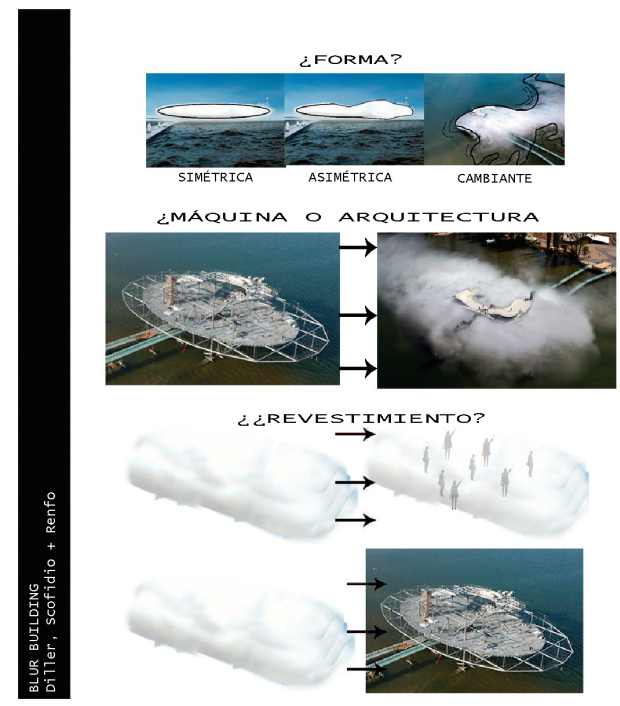

¿REVESTIMIENTO?
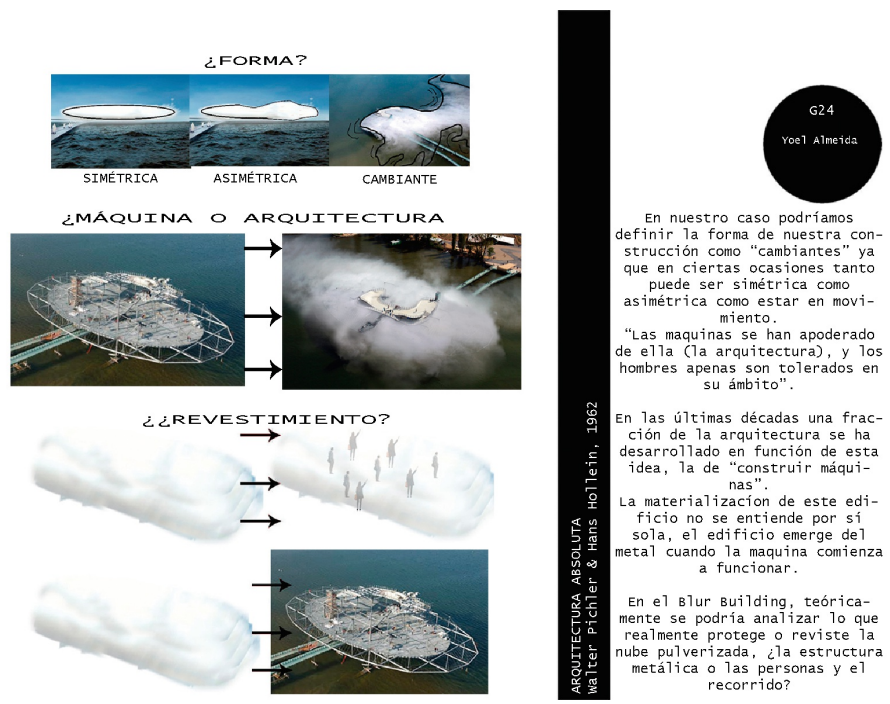

Figura 3. Análisis comparativo entre el texto "Arquitectura absoluta", de Pichler y Hollegin y el Blur Building, de Diller Scofidio + Renfo. 


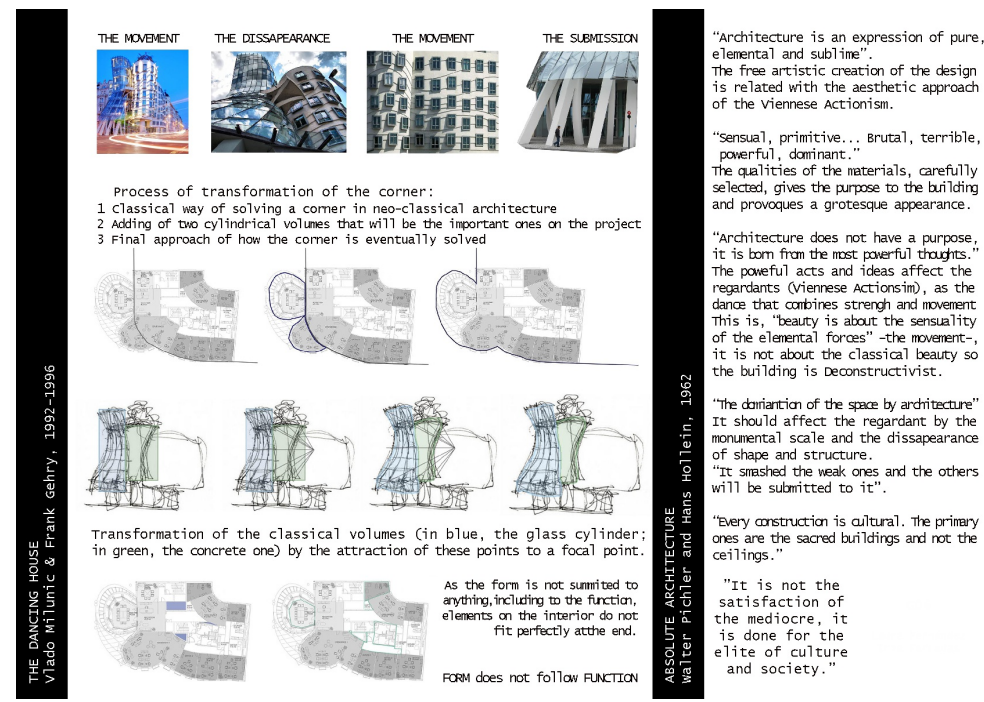

Figura 4. Análisis comparativo entre el texto "Arquitectura absoluta", de Pichler y Hollegin y la Dancing House, de Frank Gehry.

El tercer ejemplo de grupos que se enfrentan a un mismo texto consiste en analizar el "Manifiesto del enmohecimiento", contra el racionalismo en arquitectura de Hundertwasser. Se trata, por tanto, de buscar un desarrollo urbanístico basado en las ideas del manifiesto. El primero de los grupos (Figura 5) escoge como representativa la Ciudad Abierta de Valparaiso, desarrollada por la Cooperativa Amereida, en la cual identifica los conceptos principales defendidos por Hundertwasser. En el segundo caso (Figura 6), el ejemplo escogido es la Quinta Monroy planteada por el arquitecto Alejandro Aravena. Este grupo toma, además, como referente la obra pictórica de Hundertwasser, poniendo de manifiesto la diferencia formal notable existente entre ésta y la construcción de Aravena, lo cual no impide establecer los lazos conceptuales necesarios para el correcto desarrollo del ejercicio. 

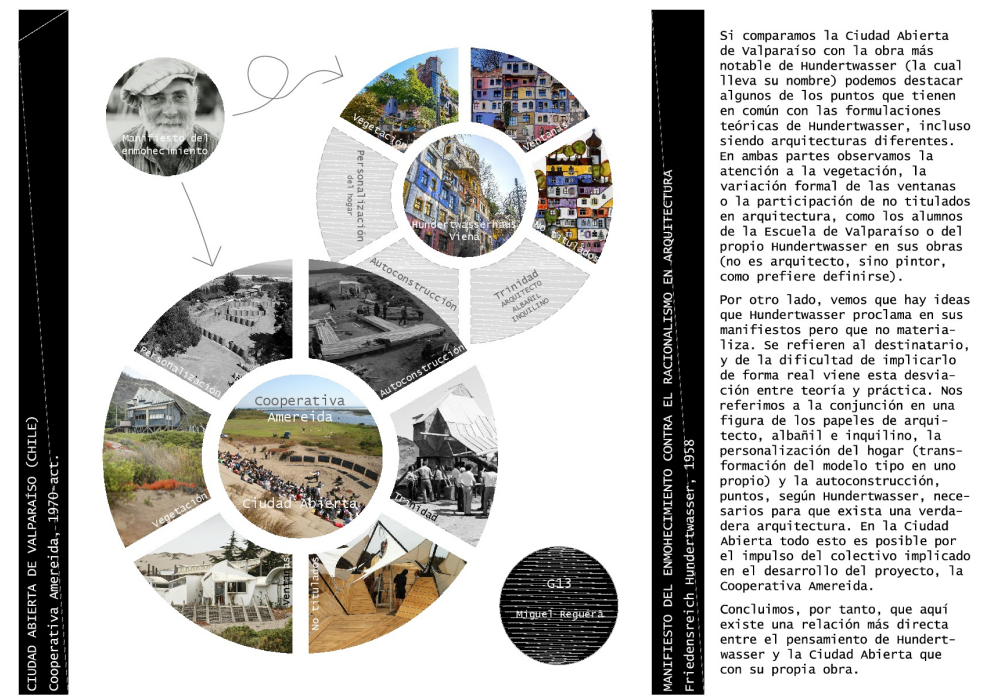

Figura 5. Análisis comparativo entre el texto de Hundertwasser y la Ciudad Abierta de Valparaiso, de Cooperativa Amereida.
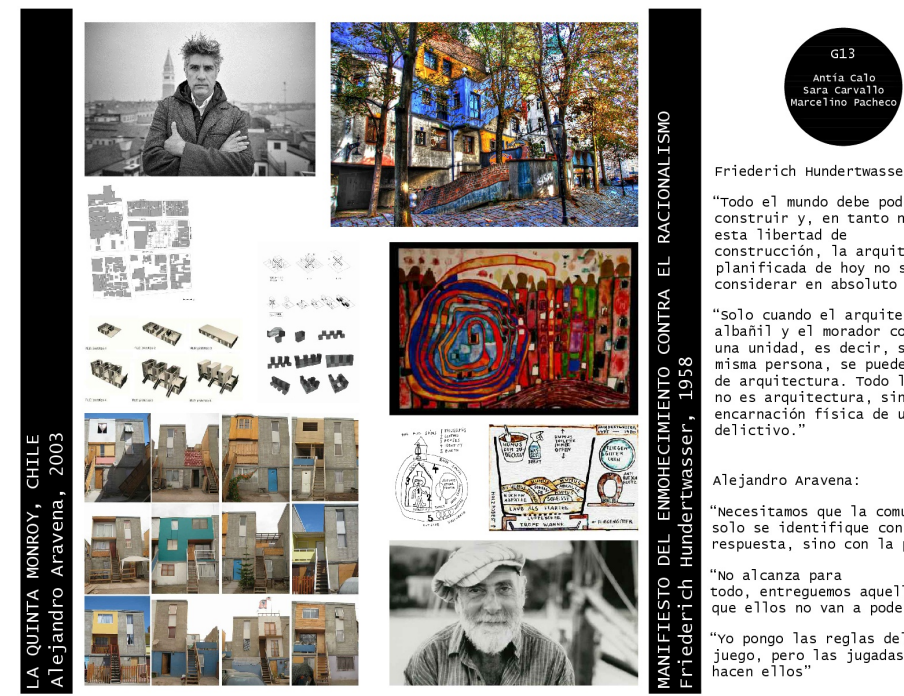

Friederich Hundertwasser:

"Todo el mundo debe poder construir $y$, en tanto no exista esta libertad de

construccion, la arquitectura
planificada de hoy no se puede planificada de hoy no se puede,
considerar en absoluto un arte"

"solo cuando el arquitecto, el albañi l y el morador constituye una unidad, es decir, son la de arquitectura rodo 70 demás no es arquitectura, sino la encarnación física de un acto delictivo."

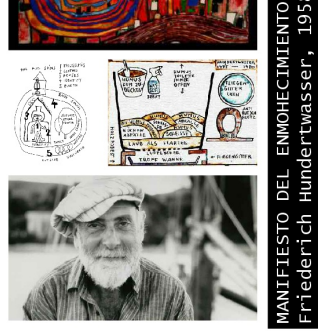

Alejandro Aravena:

Necesitamos que la comunidad no solo se identifique con la respuesta, sino con la pregunta" "No alcanza para ado, entreguemos aque11a parte Yo pongo las reglas de 1 juego, pero las jugadas las

Figura 6. Análisis comparativo entre el texto de Hundertwasser y la Quinta Monroy, de Aravena. 


\section{LOS RECURSOS DEL ESTUDIANTE DE ARQUITECTURA}

Se exponen a continuación tres trabajos en los que los diferentes recursos gráficos empleados por los alumnos resultan definitorios a la hora de establecer conclusiones.

El primero de ellos (Figura 7) busca poner en relación un extracto de un texto escrito por Hugo Häring con la Casa Estudio número 8 proyectada por los Eames. Aquí, los alumnos se centran en un aspecto concreto de la obra: el mobiliario. Así, utilizan el fotomontaje como recurso para demostrar la consonancia entre las pequeñas piezas de "arquitectura móvil" proyectadas por los mismos arquitectos y la obra de arquitectura de las contiene, planteando hipótesis en las que la casa estaría poblada de otro tipo de piezas en discordancia a la idea arquitectónica principal. En este caso resulta muy revelador el fragmento de texto que escogen como representativo de su intención: “... el director del banco actual regresa de su despacho en un Maybach y en casa se sienta en un salón Luis XVI. ¿Y dónde se debe sentar, mientras no se le proporcione el objeto equivalente a su Maybach?".
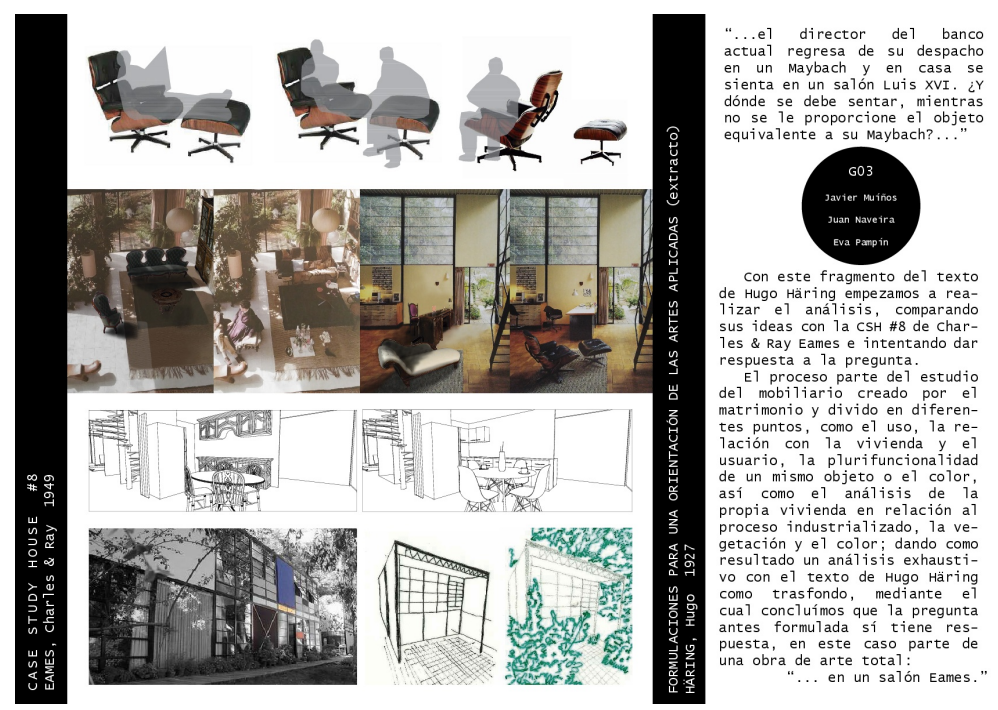

Figura 7. Análisis comparativo entre el texto "Formulaciones para una orientación de las artes aplicadas", de Hugo Häring y la 8 CSH, de Charles y Ray Eames.

En el segundo caso (Figura 8), el fotomontaje también es el protagonista del resultado analítico final. Se trata de un ejemplo excepcional en el que la fecha de construcción del edificio 
escogido es anterior al texto proporcionado por el profesorado. La intención de los alumnos con el empleo de las técnicas gráficas es imaginar cómo sería la Unidad de Habitación de Le Corbusier de haber sido construida en la época en la que se redactaron los "Diez puntos del urbanismo espacial" de Yona Friedman.
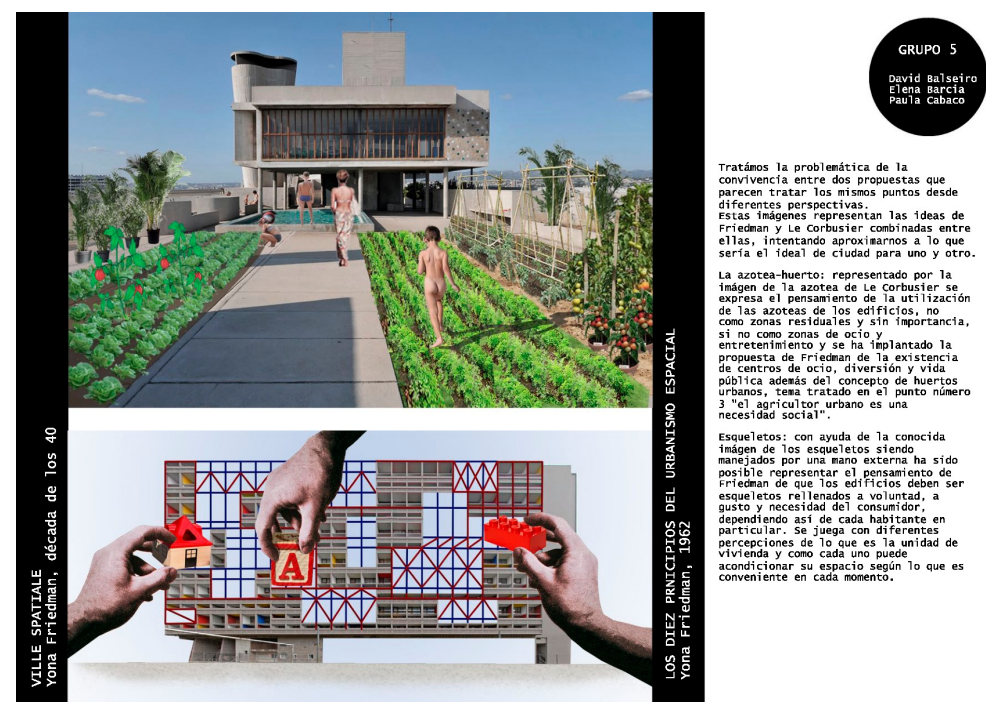

Figura 8. Análisis comparativo entre el texto "Los diez principios del urbanismo espacial”, de Yona Friedman y la Unité de Habitación, de Le Corbusier.

\section{ATENDIENDO AL CONTEXTO SOCIAL}

En este grupo de trabajos se hace especial énfasis a las condiciones sociales de la época en la que se redacta el texto que se les propone analizar.

El primer caso (Figura 9) acomete el análisis del Manifiesto futurista, si bien, al no contar con ejemplos de arquitectura construida, las alumnas se sirven de los ejemplos pictográficos correspondientes a la esta misma ideología. De este modo, se apoyan en pinturas futuristas a la hora de establecer una relación entre las ideas del manifiesto y el proyecto del Museo de arte Ordrupgaard. 


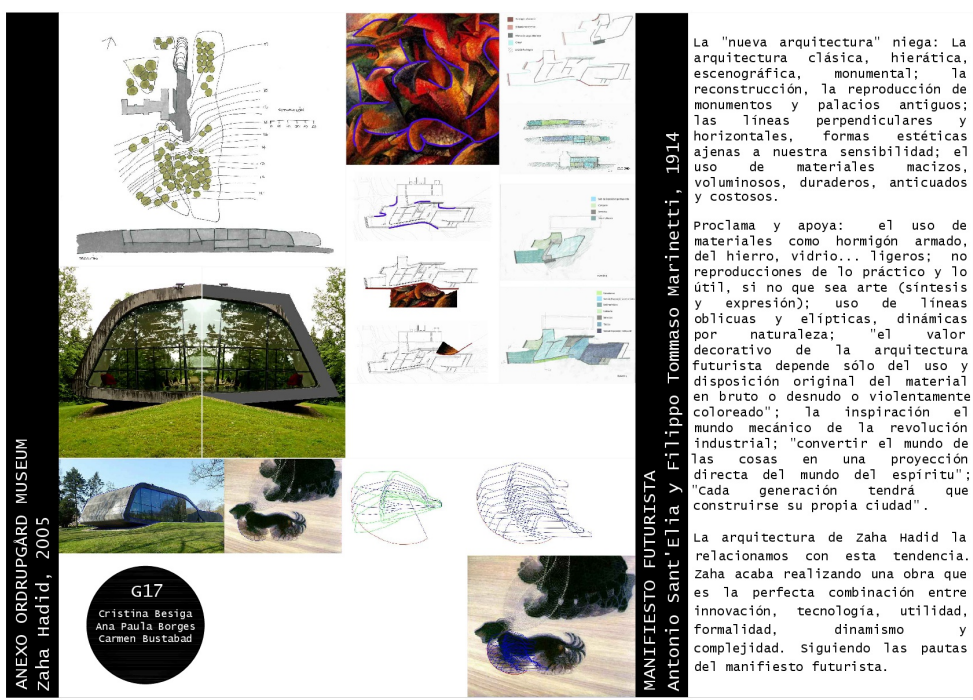

Figura 9. Análisis comparativo entre el texto "Manifiesto futurista", de Sant' Elia y Tommaso Marinetti y el Museo Ordrupgaard, de Zaha Hadid.

A la hora de seleccionar un edificio que ejemplifique las ideas expuestas en la "Tesis de la Werkbund" de 1914 redactada por Herman Muthesius, Ios alumnos fijan su atención en la construcción de los pabellones de los Comedores de la Seat de los arquitectos Ortiz-Echagüe, Barbero y De la Joya (Figura 10). Así, la estandarización propuesta en la tesis se pone en relación con la necesidad de uso de recursos disponibles en el periodo de posguerra de nuestro país. En el caso de los pabellones, se hizo uso de piezas provenientes de la industria aeronáutica a la hora de acometer el diseño de ciertas partes del edificio, que los alumnos fueron capaces de ilustrar. 

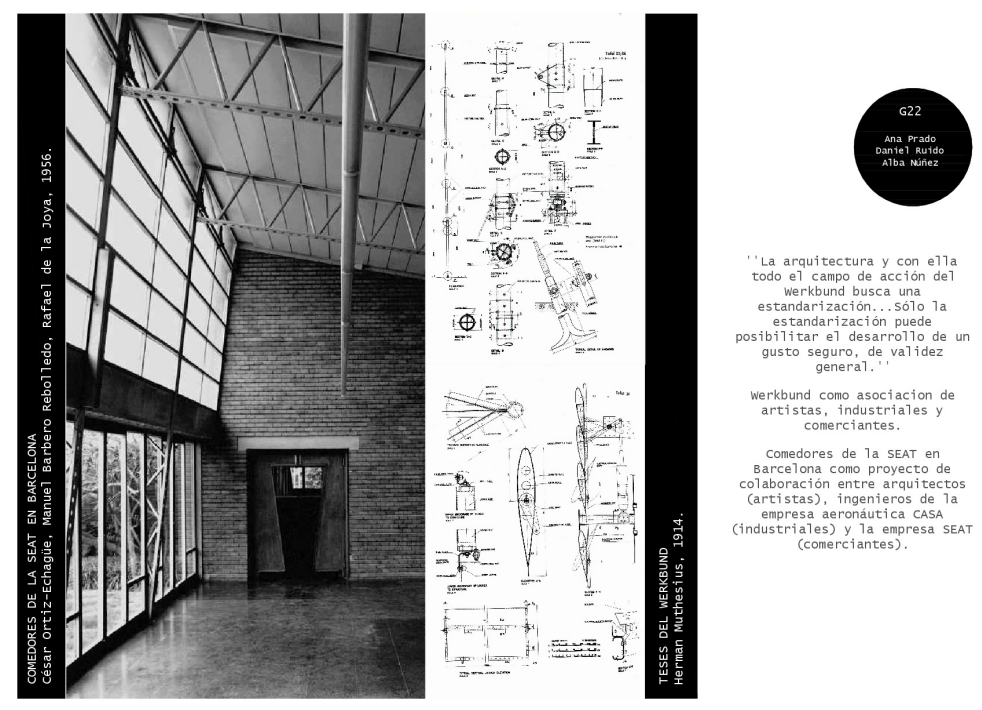

Figura 10. Análisis comparativo entre el texto "Teiss de la Werkbund", de Muthesius y los Comedores de la Seat, de Ortiz-Echagüe, Barbero y De la Joya.

El último caso aquí expuesto hace referencia a una situación similar, estableciendo una relación entre las necesidades surgidas tras la segunda guerra mundial, descritas en el Llamamiento de posguerra, y la idea de reunificación que supuso la construcción de la cúpula del Reichstag, del arquitecto Norman Foster (Figura 11). 

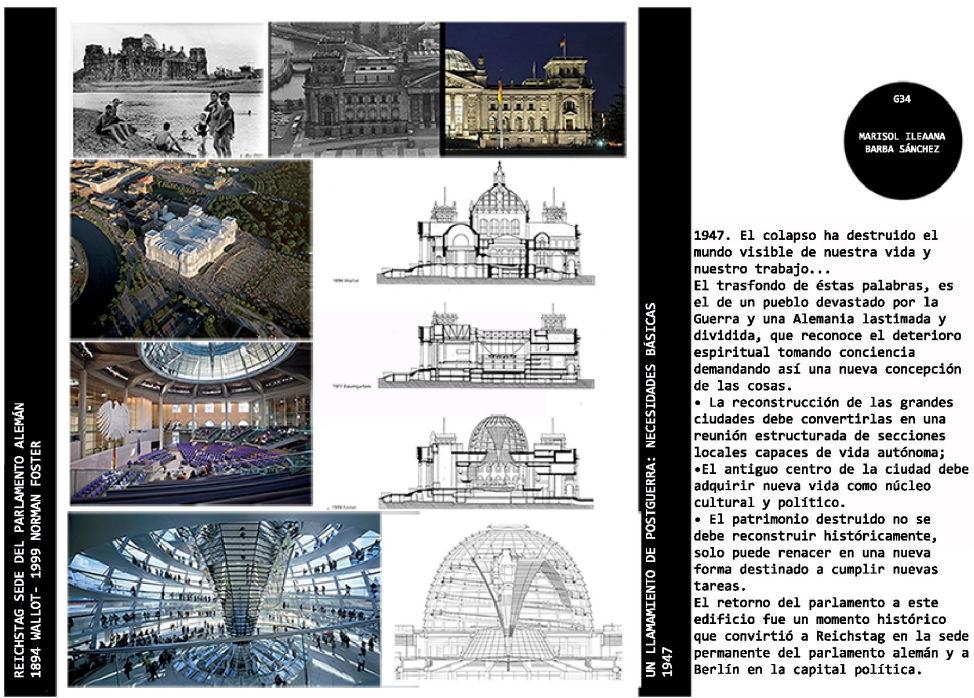

Figura 11. Análisis comparativo entre el texto "Un llamamiento de posguerra" y la cúpula del Reichstag, de Foster.

\section{CONCLUSIONES}

La experiencia presentada, si bien propone una aproximación diferente para el curso 2016-17, forma parte de una estrategia que se ha aplicado a la asignatura de Teoría de la Arquitectura desde el curso académico 2008-09. Durante este tiempo ha sido posible establecer una serie de parámetros de valoración sobre la experiencia desarrollada.

En primer lugar, y desde el primer momento, el alumnado se ha mostrado altamente receptivo a esta metodología innovadora, lo que ha alentado al profesorado a seguir planteando nuevos retos en los siguientes cursos, de forma que ha sido posible explorar diversos modos de acometer el desarrollo de esta metodología docente.

El objetivo perseguido por la asignatura, entender teoría y proyecto como aspectos inseparables, ha encontrado en la metodología utilizada en los trabajos del curso la mejor herramienta para su desarrollo.

El proceso no solamente permite al alumno establecer lazos que en un futuro le ayuden a forjar su propia idea arquitectónica sobre una base teórica sólida, sino que además les proporciona un primer contacto con una metodología de investigación. A partir de la propuesta inicial, los 
alumnos abordan la búsqueda de documentación mediante la consulta bibliográfica, el enfoque de los análisis, la elaboración de documentación gráfica y escrita para el tema tratado y un trabajo de síntesis e interpretación de conclusiones.

Esta estrategia de enseñanza finaliza con la difusión de resultados a través de una exposición, que permite visualizar a otros docentes y estudiantes este mecanismo de aprendizaje.

Además, la aportación de los trabajos no solamente ha tenido buenos resultados dentro de la propia materia en la que se desarrollan, sino que, recurriendo al área de Proyectos Arquitectónicos, se ha buscado evaluar la posible influencia de la asignatura en las propuestas creativas de los alumnos en sus trabajos proyectuales del mismo año académico, con resultados manifiestamente positivos y enriquecedores en su evolución a lo largo del curso. Se ha evaluado, de este modo, la validez de la premisa de la que se partió a la hora de establecer esta nueva metodología docente de la asignatura: la teoría como herramienta esencial en el proceso de proyecto.

\section{REFERENCIAS}

- Agrasar, F. (2008). Introducción al conocimiento de la arquitectura. A Coruña: COAG.

- Alberti, L. B. (1977). De Re aedificatoria. Valencia: Albatros.

- Eco, U. (2002). La definición del arte. Barcelona: Destino.

- Gadamer, H. G. (2013). Elogio a la teoría. Barcelona: RBA Libros.

- Giedion, S. (1957). History and the architect. Journal of Architectural Education, XII (2).

- Gregotti, V. (2000). Sulle orme di Palladio. Ragione e pratica dell'architettura. RomaBari: Laterza.

- Gregotti, V. (1993). Excerpt from Territory and Architecture. En Ockman, J. (1993). Architecture Culture 1943-1968: A Documentary Anthology. Nueva York: Rizzoli and The Trustees of Columbia University.

- Gregotti, V. (1972). El territorio de la arquitectura. Barclonea: Gustavo Gili, S. A. 
- Gregotti, V. (1993). Desde el interior de la arquitectura. Un ensayo de interpretación. Barcelona: Península.

- Hearn, M. L. (2003) Ideas that Shaped Buildings. Cambridge, Mass: The MIT Press.

- Norberg-Schulz, C. (1979). Intenciones en arquitectura. Barcelona: Gustavo Gili, S. A.

- Solá-Morales, I. (2003). Inscripciones. Barcelona: Gustavo Gili, S. A.

- Vitruvio, M. L. (1982). Los Diez Libros de la Arquitectura. Barcelona: Iberia.

- Zevi, B. (1969). Architectura in nuce. Madrid: Aguilar.

- Zevi, B. (1997). Legger, scrivere, parlare architectura. Venecia: Marsilio. 